\title{
Effects of column asymmetry on thermal diffusion separations
}

\author{
Jan P. Sørensen, Max S. Willis*, and Warren E. Stewart ${ }^{\dagger}$ \\ Department of Chemical Engineering and Mathematics Research Center, University of Wisconsin, Madison, Wisconsin 53706
}

(Received 16 March 1973)

\begin{abstract}
The theory of the Clusius-Dickel column in static operation is extended to include eccentric misalignment of the inner cylinder. Variable-property effects are included. An orthogonal expansion method is used, and a computer program is provided for general application of the method. The new theory is useful for determining alignment tolerances in commercial separation columns or in absolute measurements of $\alpha_{T}$. The theory is supported by experiments on separation of gaseous ${ }^{20} \mathrm{Ne}$ from ${ }^{22} \mathrm{Ne}$.
\end{abstract}

\section{INTRODUCTION}

The detrimental effects of column asymmetries on thermal diffusion separations were recognized in early studies of the Clusius-Dickel column. ${ }^{1,2}$ Estimates of these 'parasitic effects' were given by the foregoing authors and by Dickel, ${ }^{3}$ but the agreement with experiments was poor. ${ }^{3}$ In view of the need for an accurate theory to establish tolerances for column design, we treat the performance of ec- centric columns here in greater detail.

For asymmetric columns it is preferable to solve the equations of change directly for the temperature, velocity and composition profiles, rather than use the conventional method ${ }^{1-4}$ of partial integration. The direct approach gives a clearer phys ical picture of the interaction of the flow and diffusion phenomena, and also is easier for this threedimensional problem.

\section{NOTATION}

$A_{i j k}$

$a$

$b_{1}, b_{2}, b_{3}$

$C_{i j g}, C_{i j p}$

$c_{1}, c_{2}$

$D_{\mathrm{AA}} *(T)$

$E$

$e_{D}, e_{k}, e_{\alpha}, e_{\mu}$

$G(\eta, \xi)$

$g$

$H=H^{\prime} p^{2}$,

$K=K_{c}+K_{p}+K_{d}$

$=\left(K_{c}^{\prime}+K_{p}^{\prime}\right) p^{4}+K_{d}$

$k(T)$

L

$N_{\eta}, N_{\xi}$

$p$

$q$

$R$

$T(\eta)$
Trial function coefficients for the composition

Dimensionless $y$ coordinate of pole at $\eta=\infty$

Constants, Eq. (47)

Trial function coefficients for the velocity

Constants, Eq. (39)

Isotopic ordinary diffusivity

Dimensionless constant, Eq. (30)

Exponents for temperature dependence of physical properties

Transformation function, $(\cosh \eta-\cos \xi)^{2}$

Gravitational acceleration

Column transport coefficients

Thermal conductivity

Distance between column sampling points

Numbers of collocation points in $\eta$ and $\xi$ directions

Pressure

Column separation factor, Eq. (43)

Tube radius

Absolute temperature 


$\begin{array}{ll}u(\eta) & \text { Coordinate, Eq. (17) } \\ v_{z}(\eta, \xi) & \text { Upward velocity component } \\ V_{g}, V_{p}, V & \text { Dimensionless velocity components, Eqs. (9) and (12) } \\ x, y, z & \text { Rectangular coordinates } \\ & \text { Thermal diffusion factor } \\ \alpha_{T}(T) & \text { Dimensionless displacement of inner cylinder from tube axis } \\ \epsilon & \text { Bipolar coordinates, Eqs. (1), (2), and (3) } \\ \eta, \xi, \zeta & \text { Ratio of inner cylinder radius to tube radius } \\ \kappa & \text { Dominant non-zero eigenvalue in Eq. (33) } \\ \lambda_{1} & \text { Fluid viscosity } \\ \mu(T) & \text { Fluid density } \\ \rho(T) & \text { Mass fraction of ascending isotope } \\ \omega(\eta, \xi, \zeta) & \text { Mean surface composition, Eq. (41) } \\ \omega_{s}(\zeta) & \text { Mean surface composition, Eq. (26) } \\ \omega_{12}(\xi, \zeta) & \quad \text { Subscripts } \\ 0 & \text { Reference value for linearization of } \omega(1-\omega) \\ 1,2 & \text { Cold and hot wall } \\ B, T & \text { Bravity contribution to the velocity profile } \\ g & \end{array}$

\section{SYSTEM DESCRIPTION}

The ideal Clusius-Dickel column consists of a vertical tube of radius $R$ maintained at a uniform temperature $T_{1}$, and a coaxial rod of radius $\kappa R$ heated electrically to a higher temperature $T_{2}$. We consider here the effect of a deviation, $\epsilon R$, from coaxial alignment of the rod and tube.

The system is analyzed in bipolar coordinates $\eta, \xi, \zeta$, related to the rectangular coordinates as follows (see Fig. 1)

$$
\begin{aligned}
& x / a R=\sin \xi /(\cosh \eta-\cos \xi), \\
& y / a R=\sinh \eta /(\cosh \eta-\cos \xi), \\
& z / R=R^{3} \bar{\rho} g \zeta / \bar{D}_{\mathrm{AA}} * \bar{\mu} .
\end{aligned}
$$

The outer and inner wall are at $\eta=\eta_{1}$ and $\eta=\eta_{2}$, respectively.

We assume quasisteady operation ${ }^{1,2,5}$ with a gaseous mixture of heavy isotopes $\mathrm{A}$ and $\mathrm{A}^{*}$. The mixture velocity is purely vertical, and the temperature and velocity are considered independent of $\zeta$. The fluid properties $\mu, \rho, k, D_{\mathrm{AA}}{ }^{*}$, and $\alpha_{T}$ depend only on $T$ in a given column. The Dufour effect is small here and is omitted, as usual, ${ }^{1-5}$ to allow an uncoupled solution of the problem.

\section{SOLUTION FOR THE TEMPERATURE}

The temperature satisfies the differential equation

$$
\frac{\partial}{\partial \eta}\left(k \frac{\partial T}{\partial \eta}\right)+\frac{\partial}{\partial \xi}\left(k \frac{\partial T}{\partial \xi}\right)=0 .
$$

The solution for uniform wall temperatures at $\eta_{1}$ and $\eta_{2}$ is that $\int k d T$ depends linearly on $\eta$ and is independent of $\xi$ as well as $\zeta$. The fluid properties are correspondingly independent of both $\xi$ and $\zeta$. Then, if $k$ is a power function of $T$,

$$
k / \bar{k}=(T / \bar{T})^{e_{k}}
$$

the solution becomes

$\left(\frac{T}{\bar{T}}\right)=\left\{\left(\frac{T_{1}}{\bar{T}}\right)^{1+e_{k}}+\frac{\eta-\eta_{1}}{\eta_{2}-\eta_{1}}\left[\left(\frac{T_{2}}{\bar{T}}\right)^{1+e_{k}}-\left(\frac{T_{1}}{\bar{T}}\right)^{1+e_{k}}\right]\right\}^{1 /\left(1+e_{k}\right)}$.

Note that the reference conductivity $\bar{k}$ does not appear in the solution. 


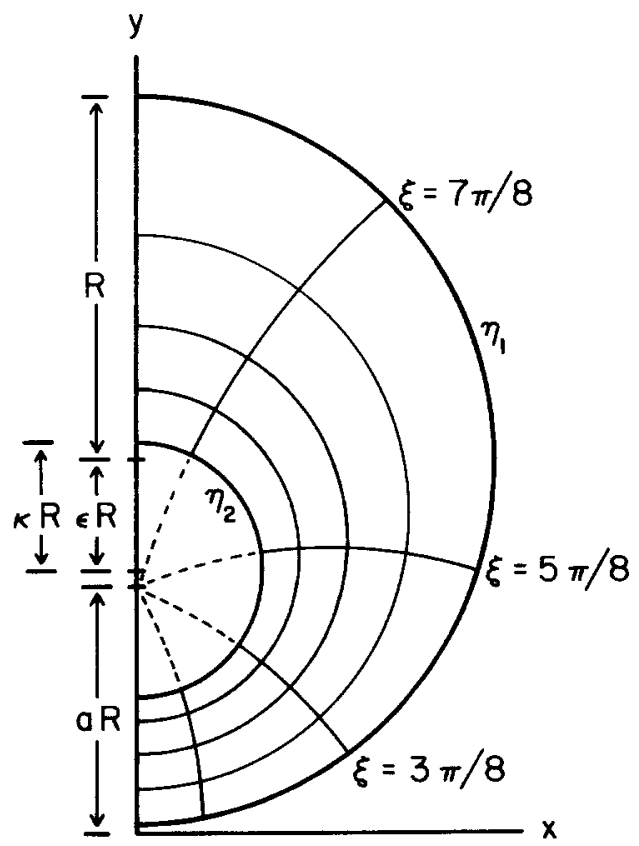

FIG. 1. Column cross section and bipolar coordinate grid.

\section{SOLUTION FOR THE VELOCITY}

In view of Eq. (6) and the assumptions previously stated, the equation of motion reduces to

$$
\left[\frac{\partial}{\partial \eta}\left(\mu \frac{\partial v_{z}}{\partial \eta}\right)+\mu \frac{\partial^{2} v_{z}}{\partial \xi^{2}}\right] \frac{G}{a^{2} R^{2}}=\frac{d p}{d z}+\rho g,
$$

where $G=(\cosh \eta-\cos \xi)^{2}$. Equation (7) is to be solved with no-slip conditions at the inner and outer walls and with a pressure gradient corresponding to zero net mass flow (static column operation)

$$
\int_{S} \rho v_{z} d S=0
$$

Here $S$ is the fluid cross section at any given value of $\zeta$. The hydrostatic solution $d p / d z=-\bar{\rho} g$ is not appropriate here, though it does hold for small temperature differences in columns with parallel plane boundaries.

We represent $v_{z}$ as the sum of a gravity term and a pressure term

$$
v_{z}=\left(\bar{\rho} g R^{2} / a^{2} \bar{\mu}\right) V_{g}+\left[\left(R^{2} / a^{2} \bar{\mu}\right)(d p / d z)\right] V_{p} .
$$

With these substitutions Eq. (7) yields the following dimensionless equations for $V_{g}$ and $V_{p}$

$$
\begin{aligned}
& \frac{\partial}{\partial \eta}\left[\frac{\mu}{\bar{\mu}} \frac{\partial V_{g}}{\partial \eta}\right]+\frac{\mu}{\bar{\mu}} \frac{\partial^{2} V_{g}}{\partial \xi^{2}}=\frac{\rho a^{4}}{\bar{\rho} G}, \\
& \frac{\partial}{\partial \eta}\left[\frac{\mu}{\bar{\mu}} \frac{\partial V_{p}}{\partial \eta}\right]+\frac{\mu}{\bar{\mu}} \frac{\partial^{2} V_{\xi}}{\partial \xi^{2}}=\frac{a^{4}}{G} .
\end{aligned}
$$

Elimination of $d p / d z$ from Eq. (9) by use of Eq. (8) gives

$$
\left(a^{2} \bar{\mu} / \bar{\rho} g R^{2}\right) v_{z}=V_{g}-V_{p} \int \rho V_{g} d S / \int \rho V_{p} d S .
$$

The functions $V_{g}(\eta, \xi)$ and $V_{p}(\eta, \xi)$ will now be determined using

$$
\begin{aligned}
& \mu / \bar{\mu}=(T / \bar{T})^{e_{\mu},} \\
& \rho / \bar{\rho}=(\bar{T} / T)
\end{aligned}
$$

as the property functions.

Each of the functions $V_{g}$ and $V_{p}$ can be represented in terms of a corresponding stream function $\psi$ as follows

$$
\rho V=\bar{\rho} G(\partial \psi / \partial \eta) \text {. }
$$

The function $\psi$ can be approximated by a finite expansion in trial functions of $\eta$ and $\xi$

$$
\psi=\sum_{i=0}^{N \eta^{-1}} \sum_{j=0}^{N-1} C_{i j} f_{i}(u) \cos (j \xi) .
$$

Here

$$
\begin{aligned}
& u=\frac{\ln \left(\eta / \eta_{1}\right)}{\ln \left(\eta_{2} / \eta_{1}\right)}, \\
& f_{0}(u)=\frac{1}{2} u^{2}-\frac{1}{3} u^{3}, \\
& f_{i}(u)=u^{i+1}(1-u)^{2} \quad i \geq 1 .
\end{aligned}
$$

The transformation in Eq. (17) was selected on the basis of numerical studies of the convergence of the approximation scheme. Equations (18) and (19) ensure satisfaction of the no-slip conditions. The cosine functions in Eq. (16) provide the required symmetry of the solution with respect to $x$.

Integration of Eq. (15) over the fluid cross section gives

$\int_{S} \rho V d S=2 \int_{\eta_{1}}^{\eta_{2}} \int_{0}^{\pi}\left(a^{2} R^{2} / G\right) \rho V d \eta d \xi=(\pi / 3) \bar{\rho} a^{2} R^{2} C_{00}$,

whence

$$
\int \rho V_{g} d S / \int \rho V_{p} d S=C_{00 g} / C_{00 p}
$$

The coefficients $C_{i j g}$ and $C_{i j p}$ are determined by collocation, that is, by requiring that Eqs. (15) and (16) satisfy Eqs. (10) and (11), respectively, at a chosen set of points in the fluid cross section of Fig. 1. This yields linear equations which can be solved numerically for the coefficients.

The departures of the approximate solutions from Eqs. (10) and (11) are made small in the mean by choosing the collocation points as Gaussian quadrature points in $(0<u<1)$ and Mehler quadrature points in $(0<\xi<\pi)$. The latter are given by

$$
\xi_{j}=\left(j-\frac{1}{2}\right) \pi / N_{\xi} \quad j=1,2 \cdots N_{\xi}
$$

and lie in the first quadrant of Fig. 1.

The convergence of the leading coefficients $C_{00 g}$ and $C_{00 p}$ with respect to $N_{\eta}$ and $N_{\xi}$ is illustrated in Table I. Three-figure convergence is achieved in 
TABLE I. Convergence of the flow coefficients .

\begin{tabular}{|c|c|c|c|c|}
\hline \multirow[b]{2}{*}{$\epsilon$} & \multirow[b]{2}{*}{$N_{\xi}$} & \multicolumn{3}{|c|}{ Values $^{2}$ of $C_{00 g}$ and $C_{00 p}$} \\
\hline & & $N_{\eta}=8$ & $N_{\eta}=10$ & $N_{\eta}=12$ \\
\hline $0^{\circ}$ & 1 & $\begin{array}{l}-0.184838 \\
-0.199933\end{array}$ & $\begin{array}{l}-0.184588 \\
-0.199771\end{array}$ & $\begin{array}{l}-0.184583 \\
-0.199768\end{array}$ \\
\hline 0.1685 & 4 & & $\begin{array}{l}-0.196825 \\
-0.209967\end{array}$ & \\
\hline & $\begin{array}{l}5 \\
6\end{array}$ & $\begin{array}{l}-0.198523 \\
-0.211082\end{array}$ & $\begin{array}{l}-0.196827 \\
-0.209969 \\
-0.196827 \\
-0.209969\end{array}$ & $\begin{array}{l}-0.196797 \\
-0.209950\end{array}$ \\
\hline 0.3370 & 4 & & $\begin{array}{l}-0.233512 \\
-0.239955\end{array}$ & \\
\hline & $\begin{array}{l}5 \\
6\end{array}$ & $\begin{array}{l}-0.236172 \\
-0.241671\end{array}$ & $\begin{array}{l}-0.234039 \\
-0.240386 \\
-0.234108 \\
-0.240441\end{array}$ & $\begin{array}{l}-0.233999 \\
-0.240363\end{array}$ \\
\hline
\end{tabular}

${ }^{a}$ The upper number in each pair is $C_{00 g}$.

${ }^{\mathrm{b}}$ Constants: $\kappa=0.08525, T_{1}=288^{\circ} \mathrm{K}, T_{2}=1098^{\circ} \mathrm{K}, \bar{T}$ $=450^{\circ} \mathrm{K}, e_{k}=0.65, e_{\mu}=0.65$.

${ }^{c}$ For this case (centered rod) the solution is axially symmetric and was done in polar coordinates.

each case with $N_{\eta} \geq 10$ and $N_{\xi} \geq 5$ (or $N_{\xi}=1$ for $\epsilon$ $=0$ ). This high accuracy is necessary since $V_{g}$ and $V_{p}$ are opposed in Eq. (12). The convergence of the remaining coefficients $C_{i j g}$ and $C_{i j p}$ is indicated by the results obtained below for the diffusion problem. The validity of the procedure is further indicated by the close agreement of $V_{p}$ in the constantproperty limit with the known exact solution. ${ }^{6,7}$

The velocity field for one of the cases in Table I is shown in Fig. 2. The asymmetry of the flow is evident.

\section{SOLUTION FOR THE COMPOSITION}

The equation of continuity for the ascending isotope is

$$
\begin{array}{r}
\rho v_{z} \frac{\partial \omega}{\partial z}=\left[\frac{\partial}{\partial \eta}\left(\rho D_{\mathrm{AA}} * \phi_{\eta}\right)+\rho D_{\mathrm{AA}} * \frac{\partial^{2} \omega}{\partial \xi^{2}}\right] \frac{G}{a^{2} R^{2}} \\
+\rho D_{\mathrm{AA}} * \frac{\partial^{2} \omega}{\partial z^{2}},
\end{array}
$$

where

$$
\phi_{\eta}=\partial \omega / \partial \eta+\omega(1-\omega) \alpha_{T}(\partial \ln T / \partial \eta) .
$$

The impermeability of the wall gives the boundary condition

$$
\phi_{\eta}=0 \text { at } \eta=\eta_{1} \text { and } \eta=\eta_{2} .
$$

The end conditions will be discussed after the general solution of these equations is indicated.

The nonlinear term $\omega(1-\omega)$ can be closely approximated by inserting the following average of the surface compositions

$$
\omega_{12}(\xi, \eta)=\frac{1}{2}\left[\omega\left(\eta_{1}, \xi, \zeta\right)+\omega\left(\eta_{2}, \xi, \zeta\right)\right] .
$$

This is a refinement of the standard practice ${ }^{1-5}$ of taking $\omega(1-\omega)$ at the cold-wall composition. Expanding this product and truncating to linear terms, we obtain

$$
\omega(1-\omega) \doteq \omega_{12}\left(1-2 \omega_{0}\right)+\omega_{0}^{2},
$$

where $\omega_{0}$ is the value of $\omega_{12}$ at a reference point on the plane $\zeta=\zeta_{0}$.

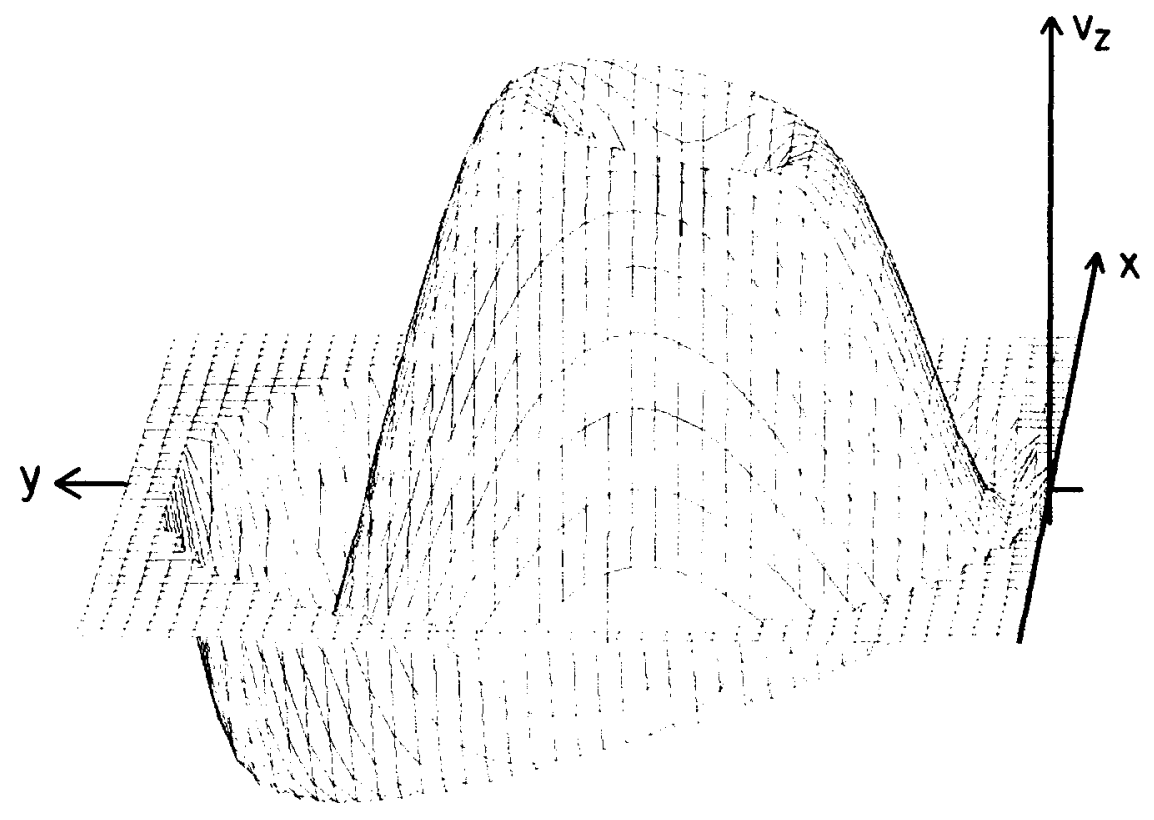

FIG. 2. Isometric view of velocity profile of $\mathrm{Eq}$. (12) for an eccentric column with $\epsilon=0.1685$. 
TABLE II. Convergence of the dominant eigenvalue.

\begin{tabular}{lllcr}
\hline$\epsilon$ & & & \multicolumn{3}{c}{ Values $^{2}$ of $1 / \lambda_{1}$} \\
& $N_{\xi}$ & $N_{\eta}=8$ & $N_{\eta}=10$ & $N_{\eta}=12$ \\
\hline 0 & 1 & 0.032608 & 0.032716 & 0.032718 \\
0.1685 & 4 & & 0.081702 & \\
& 5 & 0.082489 & 0.081772 & 0.081744 \\
& 6 & & 0.081775 & \\
0.3370 & 4 & & 0.16540 & \\
& 5 & 0.17270 & 0.17292 & 0.17290 \\
& 6 & & 0.17453 & \\
\hline \hline
\end{tabular}

a The constants of Table I are used here. In addition, $\bar{\alpha}_{T}\left(1-2 \omega_{0}\right)=0.0227, e_{D}=1.65, e_{\alpha}=0.35$, and $E=0$.

Equations (23) and (24) can now be rewritten as

$$
\begin{array}{r}
V \frac{\partial \omega}{\partial \zeta}=\frac{G}{\rho \bar{D}_{\mathrm{AA}} *}\left[\frac{\partial}{\partial \eta}\left(\rho D_{\mathrm{AA}} * \phi_{\eta}\right)+\rho D_{\mathrm{AA}} * \frac{\partial^{2} \omega}{\partial \xi^{2}}\right] \\
+\frac{D_{\mathrm{AA}}^{*}}{\bar{D}_{\mathrm{AA}}^{*}} a^{2} E^{2} \frac{\partial^{2} \omega}{\partial \xi^{2}}, \\
\phi_{\eta}=(\partial \omega / \partial \eta)+\left[\omega_{12}\left(1-2 \omega_{0}\right)+\omega_{0}^{2}\right] \alpha_{T}(\partial \ln T / \theta \eta),
\end{array}
$$

where

$$
E=\bar{D}_{\mathrm{AA}} * \bar{\mu} / R^{3} \bar{\rho} g .
$$

The solution for $\omega(\eta, \xi, \zeta)$ will be determined from Eqs. (25), (28) and (29) using

$$
\begin{aligned}
& D_{\mathrm{AA}} * / \bar{D}_{\mathrm{AA}}{ }^{*}=(T / \bar{T})^{e_{D}}, \\
& \alpha_{T} / \bar{\alpha}_{T}=(T / \bar{T})^{e_{\alpha}}
\end{aligned}
$$

as the property functions.

The general solution for $\omega$ can be approximated as closely as desired, in the neighborhood of the composition $\omega_{0}$, by taking enough terms in the following trial function

$\omega\left(1-2 \omega_{0}\right)+\omega_{0}^{2}=\sum_{k} e^{\lambda_{k}\left(\zeta-\xi_{0}\right)} \sum_{i=0}^{N_{\eta}-1} \sum_{j=0}^{N_{\xi}-1} A_{i j k} F_{i}(u) \cos (j \xi)$.

Equation (25) is satisfied for $\phi_{\eta}$ of Eq. (29) by taking the functions $F_{i}(u)$ as follows

$$
\begin{aligned}
& F_{0}(u)=1+B \frac{1-(T / \bar{T})^{e_{\alpha}}}{e_{\alpha}}, \\
& F_{1}(u)=\frac{1}{2} u^{2}-\frac{1}{3} u^{3}-\frac{1}{12}, \\
& F_{i}(u)=u^{i}(1-u)^{2} \quad i \geq 2,
\end{aligned}
$$

where

$$
B=\frac{2 \bar{\alpha}_{T}\left(1-2 \omega_{0}\right)}{2+\bar{\alpha}_{T}\left(1-2 \omega_{0}\right)\left[\left(T_{2} / \bar{T}\right)^{e_{\alpha}}+\left(T_{1} / \bar{T}\right)^{e_{\alpha}}-2\right] e_{\alpha}^{-1}} .
$$

For constant $\alpha_{T}$, Eq. (34) is rendered determinate by the substitution

$$
\lim _{e_{\alpha} \rightarrow 0}\left[\frac{1-(T / \bar{T})^{e_{\alpha}}}{e_{\alpha}}\right]=\ln (\bar{T} / T)
$$

The coefficients $A_{i j k}$ and eigenvalues $\lambda_{k}$ are found by requiring Eq. (33) to satisfy Eq. (28) at the same collocation points used in the solution for $V$. The number of eigenvalues is $N_{\eta} N_{\xi}$ if axial diffusion is neglected, and $2 N_{\pi} N_{\xi}$ if axial diffusion is included. Each value of $k$ gives an eigenfunction of the diffusion problem.

A single exponential term, $\exp \left(\lambda_{1} \zeta\right)$, suffices in Eq. (33) for all cases studied here. The higher eigenvalues are so large (at least 1000 times $\lambda_{1}$ in magnitude) that their contributions can be neglected in the usual mode of operation, which excludes the end regions of the column. As shown below, $\lambda_{1}$ is proportional to the separation accomplished per unit length of column.

The convergence of the dominant eigenvalue with respect to $N_{\eta}$ and $N_{\xi}$ is illustrated in Table II for several column geometries. The convergence becomes slower with increasing $\epsilon$, but is more than adequate for practical needs.

The eigenvalue $\lambda_{1}$ is proportional to $\left(1-2 \omega_{0}\right) \bar{\alpha}_{T}$ within terms of order $\bar{\alpha}_{T}$, e.g., within $1 \%$ for ${ }^{20} \mathrm{Ne}-{ }^{22} \mathrm{Ne}$. The composition enters the calculation of $\lambda_{1}$ only through the specification of the product $\left(1-2 \omega_{0}\right) \bar{\alpha}_{T}$.

The influence of axial diffusion on $\lambda_{1}$ is shown in Table III. The relation

$$
\bar{\alpha}_{T}\left(1-2 \omega_{0}\right) / \lambda_{1}=c_{1}+c_{2} E^{2}
$$

fits the results almost exactly, as can be seen from the final column of the table.

\section{COLUMN COEFFICIENTS}

The ratio of the column coefficients $H$ and $K$ for static separation is, by definition ${ }^{1}$

$$
H / K=\left[1 / \omega_{s}\left(1-\omega_{s}\right)\right]\left(d \omega_{s} / d z\right)
$$

in which $\omega_{s}$ is a representative composition at the given height in the column. We use

$$
\omega_{s}=(1 / \pi) \int_{0}^{\pi} \omega_{12}(\xi, \zeta) d \xi \text {. }
$$

Evaluation of $H / K$ from the dominant term of $\mathrm{Eq}$.

TABLE III. Effect of axial diffusion on the dominant eigenvalue.

\begin{tabular}{ccc}
\hline$E^{2} \times 10^{8}$ & $1 / \lambda_{1}{ }^{a}$ & $1 / \lambda_{1}-\left(1 / \lambda_{1}\right)_{E=0}$ \\
\hline 0 & 0.081744 & 0 \\
25 & 0.091225 & 0.00948 \\
50 & 0.100695 & 0.01895 \\
75 & 0.110156 & 0.02841 \\
100 & 0.119611 & 0.03787 \\
200 & 0.157389 & 0.07565 \\
\hline
\end{tabular}

${ }^{a}$ Constants are the same as in Table II except that here $E$ is varied, $\epsilon=0.1685, N_{\eta}=12$, and $N_{\xi}=5$. 
TABLE IV. Effect of column geometry and axial diffusion on the dominant eigenvalue.

\begin{tabular}{lccc}
\hline \hline \multicolumn{1}{c}{$k$} & $b_{1}{ }^{\mathrm{a}}$ & $b_{2}{ }^{\mathrm{a}}$ & $b_{3}{ }^{\mathrm{a}}$ \\
\hline 0.08525 & 0.04673 & 34800 & 2.38 \\
0.2 & 0.03545 & 39000 & 1.59 \\
0.5 & 0.02591 & 50500 & 0.914 \\
0.8 & 0.02182 & 63200 & 0.624 \\
0.9 & 0.02086 & 67500 & 0.515 \\
0.95 & 0.02042 & 69600 & 0.386 \\
0.99 & 0.02009 & 71300 & 0.31 \\
1 & $0.02001^{\mathrm{b}}$ & $71800^{\mathrm{b}}$ & \\
\hline \hline
\end{tabular}

${ }^{\text {a }}$ Computed with $N_{n}=12, N_{\xi}=5$, and the following constants: $T_{1}=288^{\circ} \mathrm{K}, T_{2}=1098^{\circ} \mathrm{K}, \bar{T}=450^{\circ} \mathrm{K}, e_{k}=e_{\mu}=0.65$, $e_{D}=1.65, e_{\alpha}=0.35, \bar{\alpha}_{T}\left(1-2 \omega_{0}\right)=0.0227$.

bobtained by linear extrapolation.

(33) then gives the predicted value

$$
H / K=\lambda_{1} E /\left(1-2 \omega_{0}\right) R \text {. }
$$

Since $\lambda_{1}$ is essentially proportional to $\left(1-2 \omega_{0}\right)$ as noted above, the calculated ratio $H / K$ is essentially independent of the mass fraction at which the linearization is performed. This is consistent with known results ${ }^{4}$ which show $H / K$ to be independent of $\omega_{s}$ for heavy isotopes in axially symmetric columns.

Integration of Eq. (40) for a column of length $L$ gives the column separation factor

$$
\ln q \equiv \ln \left[\omega_{T}\left(1-\omega_{B}\right) / \omega_{B}\left(1-\omega_{T}\right)\right]=H L / K .
$$

Equations (39), (42), and (43) give

$$
\ln q=\frac{L E \bar{\alpha}_{T} / R}{c_{1}+c_{2} E^{2}}
$$

Since $E$ varies inversely with the square of the pressure for ideal gases, Eq. (44) can be written in the form ${ }^{2}$

$$
\ln q=L H^{\prime} p^{2} /\left[\left(K_{c}^{\prime}+K_{p}^{\prime}\right) p^{4}+K_{d}\right]
$$

which shows the dependence of the separation factor on the pressure and length of column. Thus our results for $c_{1}$ and $c_{2}$ can be used to determine the ratios $\left(K_{c}^{\prime}+K_{p}^{\prime}\right) / H^{\prime}$ and $\left(K_{d} / H^{\prime}\right)$ as functions of the fluid properties and column geometry.

The solution method used here is different from that of Jones and Furry, but agrees closely with their calculations for axisymmetric columns. ${ }^{4}$ For example, the coefficients in their Eq. (85)

$$
\ln q=\frac{1.710 / p^{2}}{1+0.1628 / p^{4}}
$$

are reproduced within $0.5 \%$, and this small deviation is attributable to different definitions of $\omega_{s}$. The agreement for $K_{d} / H^{\prime}$ is particularly remarkable in view of their artificial way of handling axial diffusion. The following constants were used: $\kappa$ $=0.710, \epsilon=0, R=2.458 \mathrm{~cm}, T_{1}=300^{\circ} \mathrm{K}, T_{2}$ $=573^{\circ} \mathrm{K}, \bar{T}=436.5^{\circ} \mathrm{K}, \bar{\mu}=1.51 \times 10^{-4} \mathrm{~g} \mathrm{~cm}^{-1} \cdot \mathrm{sec}^{-1}$, $\bar{\rho}=4.47 \times 10^{-4} \mathrm{~g} \mathrm{~cm}^{-3}, p \bar{D}_{\mathrm{AA}}{ }^{*}=0.475 \mathrm{~atm} \cdot \mathrm{cm}^{2} \mathrm{sec}^{-1}$, $\bar{\alpha}_{T}=0.0077, L=730 \mathrm{~cm}, e_{k}=e_{\mu}=1, e_{D}=2$, and $e_{\alpha}$ $=0$.

\begin{tabular}{|c|c|c|c|c|c|c|}
\hline \multirow[b]{2}{*}{$\epsilon$} & \multicolumn{2}{|c|}{$\left(K_{c}^{\prime}+K_{p}^{\prime}\right) / H^{\prime}$} & \multirow{2}{*}{$\frac{\mathrm{cm} / \mathrm{atm}^{2}}{\text { exptl./predicted }}$} & \multicolumn{2}{|c|}{$K_{d} / H^{\prime}$} & \multirow{2}{*}{$\frac{\mathrm{cm} \cdot \mathrm{atm}^{2}}{\text { exptl. } / \text { predicted }}$} \\
\hline & exptl. & predicted & & exptl. & predicted & \\
\hline \multirow[t]{3}{*}{0} & 29.3 & $35.7^{a}$ & 0.82 & 38.2 & $34.0^{\mathrm{a}}$ & 1.13 \\
\hline & & $32.6^{\mathrm{b}}$ & 0.90 & & $31.0^{\mathrm{b}}$ & 1.23 \\
\hline & & $27.4^{\mathrm{c}}$ & 1.07 & & $40.2^{c}$ & 0.95 \\
\hline \multirow[t]{3}{*}{0.1685} & 68.4 & $89.1^{\mathrm{a}}$ & 0.77 & 30.5 & $30.9^{\mathrm{a}}$ & 0.99 \\
\hline & & $81.4^{b}$ & 0.84 & & $28.3^{b}$ & 1.08 \\
\hline & & $68.4^{c}$ & 1.00 & & $36.6^{\mathrm{c}}$ & 0.83 \\
\hline \multirow[t]{3}{*}{0.3370} & 127 & $188^{a}$ & 0.67 & 36.1 & $24.9^{\mathrm{a}}$ & 1.45 \\
\hline & & $172 .^{\mathrm{b}}$ & 0.74 & & $22.8^{b}$ & 1.58 \\
\hline & & $145 .^{\mathrm{c}}$ & 0.88 & & $29.5^{c}$ & 1.22 \\
\hline
\end{tabular}

The influence of $\epsilon, E$, and $\kappa$ on the column performance is illustrated in Table IV. For small displacements, $\epsilon \leq 0.04(1-\kappa)$, the dominant eigen-

TABLE V. Observed and predicted column coefficients.

aPredicted with $N_{\eta}=12, N_{\xi}=5$, and the following constants: $T_{1}=288^{\circ} \mathrm{K}, T_{2}=1098^{\circ} \mathrm{K}, \bar{T}$ $=450^{\circ} \mathrm{K}, \boldsymbol{\mu}=4.0 \times 10^{-4} \mathrm{~g} \mathrm{~cm}^{-1} \cdot \mathrm{sec}^{-1}, \bar{\rho} / p=5.47 \times 10^{-4} \mathrm{~g} \mathrm{~cm} \cdot \mathrm{cm}^{-3} \mathrm{~atm}^{-1} \bar{D}_{\mathrm{AA}}=0.97 \mathrm{~atm} \cdot \mathrm{cm}^{2}$ $\sec ^{-1}, \bar{\alpha}_{T}=0.0227, e_{k}=e_{\mu}=0.65, e_{D}=1.65, e_{\alpha}=0.35 . \bar{\alpha}_{T}$ is from Ref. 8, and other physical properties are obtained from collision integrals ${ }^{9}$ for the Lennard-Jones 12-6 potential.

${ }^{b}$ Predicted as in Footnote a except that $\bar{\alpha}_{T}=0.0248$ as determined by a least-squares fit of these experiments. The measured values of $q$ are fitted with a standard error of $14 \%$.

${ }^{c}$ Predicted as in Footnote a except that $\bar{\alpha}_{T}=0.0238$ and $p \bar{\mu} \bar{D}_{\mathrm{AA}^{*}}=4.82 \times 10^{-4} \mathrm{~atm} \cdot \mathrm{g} \cdot \mathrm{cm} \mathrm{sec}^{-2}$ as determined by a least-squares fit of these experiments. The measured values of $q$ are fitted with a standard error of $5.7 \%$. 
value $\lambda_{1}$ is fitted within $1 \%$ by the following expres sion

$$
1 /(1-\kappa)^{4} \lambda_{1}=b_{1}+b_{2} E^{2} /(1-\kappa)^{6}+b_{3} \epsilon^{2} /(1-\kappa)^{2} \text {. }
$$

The corresponding behavior of $q$ can be determined by insertion of this expression into Eqs. (42) and (43). Over this small range of displacements, the axial diffusion contribution is negligibly affected by misalignment, in accord with the assumptions of Jones and Furry. ${ }^{2}$

Equation (47) and Table IV show that very close alignment is necessary when the gap between the rod and the tube is small. The results shown here are for gaseous ${ }^{20} \mathrm{Ne}-{ }^{22} \mathrm{Ne}$, but indicate the expected magnitude of the misalignment effects for other systems.

\section{COMPARISON WITH EXPERIMENTS}

The effect of column misalignment was studied experimentally ${ }^{8}$ by conducting static separations of ${ }^{20} \mathrm{Ne}-{ }^{22} \mathrm{Ne}$ mixtures at pressures from 500 to 1500 torr in a length $L=152.4 \mathrm{~cm}$ of a column with radii $R=0.9430 \mathrm{~cm}$ and $\kappa R=0.08039 \mathrm{~cm}$. Experiments were done with the wire centered in the tube $(\epsilon=0)$ and also with displacements $\epsilon R=0.15875$ and $0.3175 \mathrm{~cm}$ of the wire from the tube axis. The tube and wire were maintained at 288 and $1098{ }^{\circ} \mathrm{K}$.

Column coefficients $\left(K_{c}^{\prime}+K_{p}^{\prime}\right) / H^{\prime}$ and $K_{d} / H^{\prime}$ were determined by plotting the data according to Eq. (45). The results are shown in Table V. For comparison, predicted values are also given based on the constants of Eq. (44).

The agreement is reasonable in view of the uncertainties of the experimental parameters $\left(K_{c}^{\prime}+K_{p}^{\prime}\right) / H^{\prime}$ and $K_{d} / H^{\prime}$. The best measure of agreement is that of the separation factors $q$, which are fitted within $5.7 \%$ in Case c, using reasonable property values.

\section{CONCLUSION}

The theory developed here provides a detailed description of the effects of column eccentricity on thermal diffusion separations. The resulting loss of separation capacity is most serious for large values of $\kappa$ (small radial clearances) as shown in Table IV. For small $\kappa$ values the effect is less im- portant.

For small displacements, $\epsilon$, of the rod from the axis of the tube, the loss of separation power varies as $\epsilon^{2} /(1-\kappa)^{2}$. Thus, with a thin, well aligned center rod, it should be possible to determine accurate values of $\bar{\alpha}_{T}$ and $\bar{\mu} \bar{D}_{\mathrm{AA}} *$ from measurements of $q$ at various pressures. The recent results of Rutherford ${ }^{10}$ are in accord with this.

A computer program has been provided for general use of our solution method. See NAPS Document No. 2146 for 21 pages of supplementary material. Order from ASIS/NAPS c/o Microfiche Publications, 305 E. 46th St., New York, N.Y. 10017. Remit in advance for each NAPS accession number $\$ 1.50$ for microfiche or $\$ 5.00$ for photocopies. Make checks payable to Microfiche Publications.

\section{ACKNOWLEDGMENT}

This work was sponsored by the National Science Foundation under Grants G67242 and GK17860, and by the United States Army under contract No.: DA-31-124-ARO-D-462. The data were obtained at Mound Laboratory, Miamisburg, Ohio, operated for the U.S. Atomic Energy Commission by Monsanto Research Corporation.

*Present address: Department of Chemical Engineering,

University of Akron, Akron, Ohio 44325 .

${ }^{\dagger}$ To whom correspondence should be sent.

'W. H. Furry, R. C. Jones, and L. Onsager, Phys. Rev. 55, 1083 (1939).

${ }^{2}$ R. C. Jones and W. H. Furry, Rev. Mod. Phys. 18, 151 (1946).

${ }^{3}$ G. Dickel, Z. Naturforsch. A 16, 755 (1961)

${ }^{4}$ W. H. Furry and R. C. Jones, Phys. Rev. 69, 459 (1946).

${ }^{5}$ J. Bardeen, Phys. Rev. 58, 94 (1940).

${ }^{6}$ J. Caldwell, J. Roy. Tech. Coll. (Glasgow) 2, 203 (1930).

${ }^{7}$ N. A. V. Piercy, M. S. Hooper, and H. F. Winny, Philos. Mag. 15, 647 (1933).

${ }^{8}$ J. F. Eichelberger, G. R. Grove, and L. V. Jones, Mound Laboratory Progress Report for September 1965, USAEC Research and Development Report MLM-1299, p. 29; October 1965, MLM-1300, p. 20.

${ }^{9}$ J. O. Hirschfelder, C. F. Curtiss, and R. B. Bird, Molecular Theory of Gases and Liquids (Wiley, New York, 1954).

${ }^{10}$ W. M. Rutherford, J. Chem. Phys. 54, 4542 (1971). 